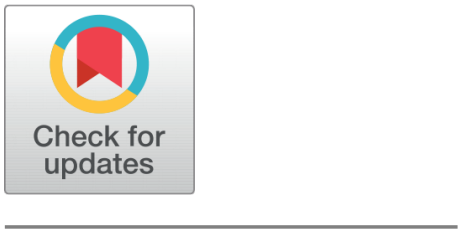

Open access

Received: 13-06-2020

Accepted: 19-07-2020

Published: $31-07-2020$

Editor: Dr. Natarajan Gajendran

Citation: Shakil M, Rashid Z, Hussain GA, Umer F (2020) Power Flow Analysis and Optimization in Ring Distribution Network of Bahawalpur using Newton Raphson Method. Indian Journal of Science and Technology 13(27): 2720-2732. https ://doi.org/10.17485/IJST/v13i27.916

* Corresponding author.

Zeeshan Rashid

Department of Electrical Engineering, The Islamia University of Bahawalpur, 63100, Pakistan. Tel.: +92-62-925-5576 zeeshan.rashid@iub.edu.pk

Funding: None

Competing Interests: None

Copyright: ( 2020 Shakil, Rashid, Hussain, Umer. This is an open access article distributed under the terms of the Creative Commons Attribution License, which permits unrestricted use, distribution, and reproduction in any medium, provided the original author and source are credited.

Published By Indian Society for Education and Environment (iSee)

\section{Power Flow Analysis and Optimization in Ring Distribution Network of Bahawalpur using Newton Raphson Method}

\author{
Muhammad Shakil ${ }^{1}$, Zeeshan Rashid ${ }^{1}{ }^{*}$, Ghulam Amjad Hussain ${ }^{2}$, \\ Farhana Umer ${ }^{1}$ \\ 1 Department of Electrical Engineering, The Islamia University of Bahawalpur, 63100, \\ Pakistan. Tel.: +92-62-925-5576 \\ 2 Department of Electrical Engineering, College of Arts and Sciences, American University of \\ Kuwait, Safat, 10002, Kuwait
}

\section{Abstract}

Background: The primary motive of an electrical power system is to generate and supply electric power efficiently and reliably to the consumer-end. Transmission losses, system instability and increasing cost in proportion to demand are the main challenges faced in this process. Power flow analysis is required to robustly predict the active / reactive power within the buses, voltage magnitude / phase angles at each bus, cost of transmission and losses well before the practical installation of the power network. Methods/Statistical Analysis: In this paper, we employ power flow analysis using Newton Raphson method and Fast Decoupled method to minimize cost of the electricity and finding optimum active and reactive powers without affecting the voltage regulation. The power flow algorithms are applied for solving the aforementioned load flow problem for ring distribution network of Bahawalpur. We carried out the modeling by obtaining realistic data for constructing bus admittance matrix and specifications of generation units and loads which are connected at the buses. Findings: As a result, optimum flow of power along with the voltage values among different regions of Bahawalpur is obtained. The results from both the algorithms successfully converge and there is an absolute match to validate the accuracy. Novelty: These novel results are of paramount importance since the proposed architecture of Bahawalpur is ring distribution network to replace the existing radial network for improved performance. Furthermore, this research will pave the way for power system planning of Bahawalpur region where all the electrical parameters are known beforehand to design the components according to the requirement.

Keywords: Electric power system; voltage magnitude; phase angle; power flow analysis; Newton Raphson method; ring distribution network; active power; reactive power 


\section{Introduction}

The prime objective of the contemporary electrical power network is continued fulfillment of the power demand utilized by all the users. It becomes a complex issue in power transmission study. Fluctuations in load, generation, lines or interconnections give rise to problems such as system instability and system losses.

By reverting to the load flow analysis and related studies, many of these critical aspects in power system applications can be evaluated to a great degree quite efficiently. Load study proves to be a critical control task in steady and effective functioning of power grids. The principle aim of the load study becomes ascertaining the steady-state working specifications in the existing electric network as well as scheduling and designing the future expansion of the network. We can find steady-state by calculating angles and magnitude of voltage, as well as the flow of reactive and active power in the system for the given loading conditions set. Here, we codify Newton Raphson technique for an optimum load flow analysis employing ring distribution system in and around the Bahawalpur area. It employs five buses, two generating and three load buses.

We have the following sections in this paper: Literature review has been discussed in section 2 in which we have presented different solution techniques for power flow problem, starting from the earliest techniques to the recent ones. This section is followed by the proposed methodology i.e. Newton Raphson algorithm in section 3. Results and conclusions have been presented in sections 4 and 5 respectively.

\section{Literature Review}

For sorting out load flow equations, different techniques are presented over the years. The numerical methodologies and loop equations employing Gauss-type solutions were basis of the early techniques ${ }^{(1)}$. The system engineers had to designate the network loops in advance so, this method proved burdensome. Upgraded approaches spotted the development of the nodal analysis in favor of the loop analysis that lead towards significantly reducing the data formation. Nonetheless, accuracy to the convergence still remained primary issue. The acceleration factors in the Gauss Seidel method were developed with the further progression in the field. The features like easy to understand, least storage requirements, easy to codify in format of computer programs are main attraction of the present generation of load flow methodologies. When applied to the solution of reasonable size of systems, these algorithms show poor convergence properties resulting in the only limitation of the method. Load flow solutions that were based upon the bus impedance matrix were shortly tested but at that time, the issues with speed and computer storage came out to be unconquerable problems. In the early 1970s, the Newton Raphson methodology and derived formulations were introduced to overcome such constraints, and it has since become very popular technique all over the power system industry ${ }^{(2)}$.

Stott and Alsac's later work rendered Newton's Fast Decoupled methodology ${ }^{(3)}$. This approach provides extremely fast, simple and very reliable power flow solution. The convergence performance of this method is poor for most of the distribution networks, although it worked well for transmission networks. This is because of its large R/X ratio that degrades Jacobian matrix's diagonal dominance.

Numerous other forms of approaches were proposed for this purpose. For example, Modified Newton Method and Modified Gauss-Seidel algorithm ${ }^{(4-8)}$ etc. Tripathy ${ }^{(9)}$ et al. introduced a technique resembling to Newton's methodology of sorting out unconditioned power networks but could not be used effectively to measure maximal power flow. Baran and Wu suggested a technique in solution of radial power flow to evaluate optimum problem of capacitor sizing ${ }^{(10)}$. The computational efficiency is increased in this process. Chiang et al. suggested three techniques in the resolution of radial system based on the Baran and $\mathrm{Wu}$ system $^{(11)}$. Goswami and Basu suggested the direct methodology in solving networks of radial and meshed distribution ${ }^{(12)}$. It also works well for heavy loads. A new power-flow method was proposed by Jasmon and Lee to obtain the solution of radial systems ${ }^{(13)}$. Using the power convergence technique, Das et al. suggested the new method of power-flow analysis by calculation of net reactive and real powers supplied by any bus for the solution of radial distribution systems ${ }^{(14)}$. To solve radial distribution networks, primary assumption was put forward as the zero initial power loss. It saves the computer storage to a great extent. Haque et al. introduced another effective methodology with more than one feeding node for resolving both meshed and radial networks ${ }^{(15)}$. This approach has excellent radial network convergence. In radial distribution networks, Eminoglu and Hocaoglu et al. suggested the simple and efficient technique for solution of load flow problem, taking into account static load voltage dependence and line charging efficiency ${ }^{(16)}$. Prasad et al. suggested the branch-current calculation technique in radial network ${ }^{(17)}$. It takes advantage of a network's tree-structure property. Ghosh and Sherpa et al. developed a system for radial distribution network power-flow solution with minimal data planning ${ }^{(18)}$. Sivanagaraju et al. suggested a distinctive technique for power-flow solution that is employed to analyze weakly meshed system using branch current matrix ${ }^{(19)}$. Kumar and Arvindhababu et al. proposed a power flow solution approach for achieving efficient convergence in distribution systems ${ }^{(20)}$. For study of weakly meshed or radial systems, Augugliaro et al. suggested a technique providing the voltage dependent demand ${ }^{(21)}$. 
At each step, impedances simulate the loads and furthermore, the solution procedure is iterative in nature. For the solution of radial distribution systems, Kaur et al. has suggested a new power-flow technique using sequential numbering scheme ${ }^{(22)}$. Suggesting a technique, with less computation and reducing data preparation, is the aim of this methodology. Sharma et al. has investigated a couple of new network reconfiguration schemes in introducing two new effective power flow techniques, and presenting simulation test results ${ }^{(23)}$. Parasher et al. showed a method for radial distribution network power-flow solution ${ }^{(24)}$. The author endeavored to reduce the data preparation in some cases, and for some other situations, the author employed data as it is without reducing its preparation.

\subsection{A Comparison between Newton-Raphson and Fast-Decoupled Methods}

We have implemented data in both Newton Raphson (NR) and Fast Decoupled (FD) algorithms in MATLAB. The results obtained thus are the same hence, the choice of a single methodology between the two might become challenging as both techniques have some advantages and disadvantages over the other. However, this selection of method might become easier if certain priority points are already kept in mind and the comparison is then drawn between the two. Following is a brief comparison between NR and FD load flow algorithms:

\begin{tabular}{ll}
\hline \multicolumn{1}{c}{ Newton-Raphson } & \multicolumn{1}{c}{ Fast-decoupled } \\
\hline $\begin{array}{l}\text { For both small and large networks, highly accurate solution can be obtained in } \\
\text { just two to three iterations. }\end{array}$ & $\begin{array}{l}\text { The number of iterations is always greater as } \\
\text { compared to NR method. }\end{array}$ \\
$\begin{array}{l}\text { The overall cost in solving power flow problem is less. } \\
\text { Large computer memory is required. }\end{array}$ & The cost is greater than that of NR methodology. \\
Fewer number of iterations result in less computational time. & Computer storage requirements are smaller. \\
Convergence is slower as compared to FD method. & Computational time is larger. \\
\hline
\end{tabular}

The NR algorithm executes results in only 6 iterations while FD algorithm takes 27 iterations. In this study, the factors of our concern are mainly economy and computational time. As the overall cost in NR algorithm is smaller than FD method and it has less number of iterations as compared to FD method, so we have preferred NR method to FD method.

\section{Proposed Methodology}

Newton Raphson algorithm is utilized in this paper for the solution of load flow problem in ring distribution network. It has been elaborated in depth below:

\subsection{Computational procedure}

For polar coordinates, the Newton Raphson Computational procedure in power flow analysis is as follows:

1. $\mathrm{Y}_{\text {bus }}$ is formed using series line impedances or admittances.

2. Initial values for phase angles and bus voltages will be supposed for all load buses, and phase angles for generator buses. Usually, these assumed values will be maintained equal to slack, reference or swing bus voltage magnitude and angle.

3. For each load bus, $\mathrm{P}$ and $\mathrm{Q}$ will be computed employing, respectively, Eqs. (38) and (39).

4. Eqs. (42) and (43) are used to compute scheduled errors $\Delta \mathrm{P}$ and $\Delta \mathrm{Q}$. Although the exact value of $\mathrm{Q}$ is not specified in case of PV buses, its limits are known. So, there are two situations before us; if calculated value of Q is within limits, there is no need to calculate $\Delta \mathrm{Q}$. But in case value of $\mathrm{Q}$ exceeds the limit, we set an appropriate limit and subtract the calculated value of $Q$ from it and the bus is treated as PQ bus.

5. Jacobian matrix is formed by computing its elements one by one.

6. $\Delta \mathrm{V}$ and $\Delta \theta$ are calculated using Eq. (44).

7. Voltage angles and magnitudes are updated using values of $\Delta \mathrm{V}$ and $\Delta \theta$ found in step 6, Eqs (54) and (55) are employed in this computation. Next iteration is started from step 2, utilizing the updated values of $\mathrm{V}$ and $\theta$.

8. This process is continued until scheduled errors $\Delta \mathrm{P}$ and $\Delta \mathrm{Q}$ are within specified tolerance for all the load buses.

$$
\Delta P_{i}^{(r)}<\varepsilon, \Delta Q_{i}^{(r)}<\varepsilon
$$


$\epsilon$ is tolerance level.

Lastly, line power flows and slack bus power is calculated.

\subsection{The Bus admittance matrix $\mathbf{Y}_{b u s}$}

Consider a small-scale power system network ( Figure 1 ) containing three lines, two generators, one load and a static capacitor attached to load bus 3 . We will suppose that network is symmetrical and operating under balanced conditions.

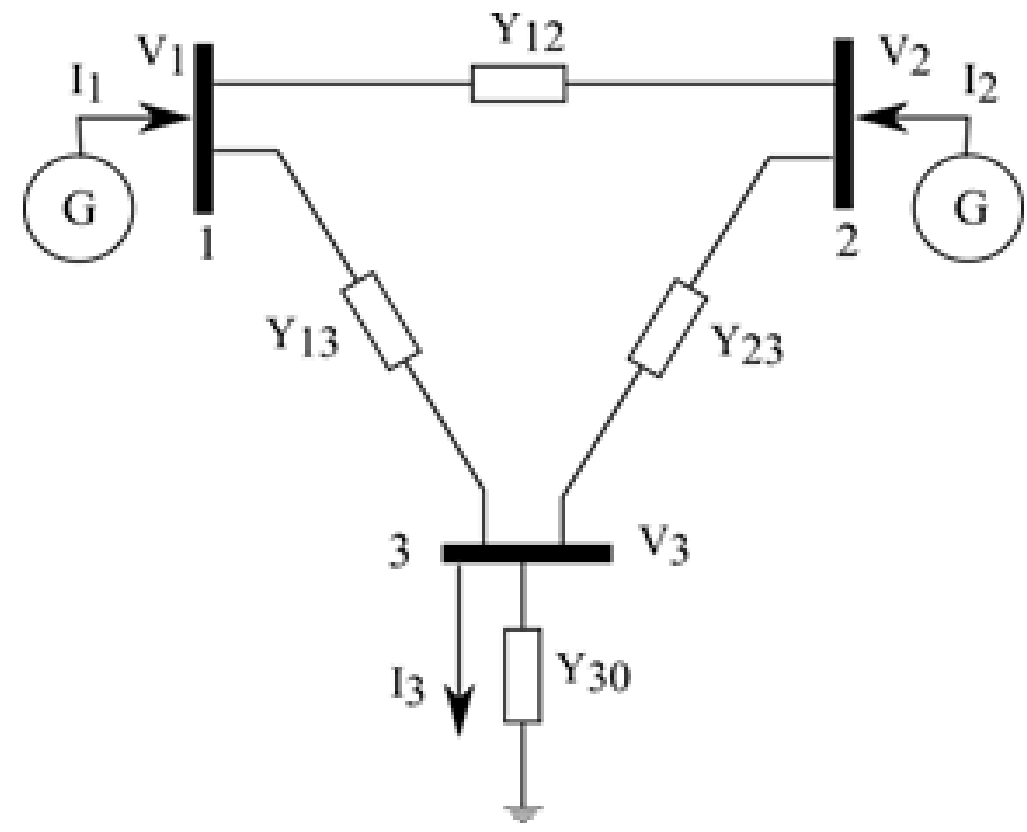

Fig 1. Power system network.

We are concerned about the steady state solution of the network shown in Figure 1 consisting of a three-node network. We can write the bus voltage equations as

$$
\begin{gathered}
I_{1}=\left(y_{12}+y_{31}\right) V_{1}-y_{12} V_{2}-y_{31} V_{3} \\
I_{2}=-y_{12} V_{1}+\left(y_{12}+y_{23}\right) V_{2}-y_{23} V_{3} \\
-I_{3}=-y_{31} V_{1}-y_{23} V_{2}+\left(y_{31}+y_{23}+y_{30}\right) V_{3}
\end{gathered}
$$

Where $y_{12}=\frac{1}{z_{12}}, y_{23}=\frac{1}{z_{23}}, y_{31}=\frac{1}{z_{31}}$.

In matrix form

$$
\left[\begin{array}{c}
I_{1} \\
I_{2} \\
-I_{3}
\end{array}\right]=\left[\begin{array}{ccc}
y_{12}+y_{31} & -y_{12} & -y_{31} \\
-y_{12} & \left(y_{12}+y_{23}\right) & -y_{23} \\
-y_{31} & -y_{23} & \left(y_{31}+y_{23}+y_{30}\right)
\end{array}\right]\left[\begin{array}{l}
V_{1} \\
V_{2} \\
V_{3}
\end{array}\right]
$$

It is notable that the negative sign indicates that the current is extracted while the positive sign indicates that the currents injected. The above equations can be written as

$$
\left[\begin{array}{c}
I_{1} \\
I_{2} \\
-I_{3}
\end{array}\right]=\left[\begin{array}{lll}
Y_{11} & Y_{12} & Y_{13} \\
Y_{21} & Y_{22} & Y_{23} \\
Y_{31} & Y_{32} & Y_{33}
\end{array}\right]\left[\begin{array}{l}
V_{1} \\
V_{2} \\
V_{3}
\end{array}\right]
$$


Where $Y_{11}=y_{12}+y_{31} ; Y_{22}=y_{12}+y_{23} ; Y_{33}=y_{31}+y_{23}+y_{30}$

and $Y_{12}=Y_{21}=-y_{12} ; Y_{23}=Y_{32}=-y_{23} ; Y_{31}=Y_{13}=-y_{31}$

The elements $\mathrm{Y}_{11}, \mathrm{Y}_{22}, \mathrm{Y}_{33}$ making the diagonal entries are called self admittances. The self admittance of a bus $\mathrm{x}$ can be found by adding all the admittances at that bus. As a general rule in bus admittance matrix, the diagonal element $\mathrm{Y}_{p p}$ can be calculated by adding all the admittances attached at bus p. i.e.,

$$
Y_{p p}=y_{p 1}+y_{p 2}+\cdots+y_{p n}
$$

Where $\mathrm{Y}_{p q}$ is the admittance of the element attached between the buses $\mathbf{p}$ and $\mathbf{q}$.

The elements $\mathrm{Y}_{12}, \mathrm{Y}_{13}, \mathrm{Y}_{21}, \mathrm{Y}_{23}, \mathrm{Y}_{31}, \mathrm{Y}_{32}$ making the off-diagonal entries are termed as mutual admittances.

$$
Y_{12}=Y_{21}=-y_{12} ; Y_{23}=Y_{32}=-y_{23} ; Y_{31}=Y_{13}=-y_{31}
$$

It is notable that all mutual admittance entries have a negative sign. As a general rule in bus admittance matrix, the off-diagonal element equals negative of the admittance attached at the nodes $\mathrm{p}$ and q. i.e.,

$$
Y_{p q}=-y_{p q}
$$

For a network having $\mathrm{n}$ buses excluding ground, a set of following equations, one for each bus, might be expressed as

$$
I_{1}=Y_{11} V_{1}+Y_{12} V_{2}+\cdots+Y_{1 n} V_{n}
$$

$$
I_{2}=Y_{21} V_{1}+Y_{22} V_{2}+\cdots+Y_{2 n} V_{n}
$$

$$
I_{n}=Y_{n 1} V_{1}+Y_{n 2} V_{2}+\cdots+Y_{n n} V_{n}
$$

In matrix form as

$$
\left[\begin{array}{c}
I_{1} \\
I_{2} \\
\vdots \\
I_{n}
\end{array}\right]=\left[\begin{array}{cccc}
Y_{11} & Y_{12} & \cdots & Y_{1 n} \\
Y_{21} & Y_{22} & \cdots & Y_{2 n} \\
\vdots & \vdots & \vdots & \vdots \\
Y_{31} & Y_{32} & \cdots & Y_{n n}
\end{array}\right]\left[\begin{array}{c}
V_{1} \\
V_{2} \\
\vdots \\
V_{n}
\end{array}\right]
$$

In a more compact form

$$
\begin{gathered}
I_{\text {bus }}=Y_{\text {bus }} V_{\text {bus }} \\
\text { Where } I_{\text {bu s }}=\text { bus current vector }=\left[\begin{array}{l}
I_{1} \\
I_{2} \\
\vdots \\
I_{n}
\end{array}\right] \\
V_{\text {bus }}=\text { bus voltage vector }=\left[\begin{array}{l}
V_{1} \\
V_{2} \\
\vdots \\
V_{n}
\end{array}\right]
\end{gathered}
$$




$$
Y_{\text {bus }}=\text { bus admittance matrix }=\left[\begin{array}{cccc}
Y_{11} & Y_{12} & \cdots & Y_{1 n} \\
Y_{21} & Y_{22} & \cdots & Y_{2 n} \\
\vdots & \vdots & \vdots & \vdots \\
Y_{31} & Y_{32} & \cdots & Y_{n n}
\end{array}\right]
$$

With reference to ground, we calculate the nodal voltages, while Eq. (10) is a vector equation comprising n scalar equations. It is called the nodal current equation. If the power system elements have mutual coupling, the bus admittance matrix cannot be developed directly by examining the one line diagram. In existence of mutual coupling between power system elements, the inspection method flops. In such a case, $\mathrm{Y}_{\text {bus }}$ can be formed from graph theoretic approach. However, the mutual coupling between power system elements is only present in the case of transmission lines going parallel for a long distance. But this coupling is also weak. Therefore, the mutual coupling can be disregarded for all practical purposes and $\mathrm{Y}_{\text {bus }}$ is formulated by inspection method.

\subsection{The Newton-Raphson algorithm}

Owing to its powerful convergence characteristics, the Newton Raphson (NR) methodology has come out as the most fruitful technique in the large-scale load flow analysis. Iteration approach is used by this technique to resolve a set of nonlinear-algebraic equations, as shown below:

$$
\left.\begin{array}{l}
f_{1}\left(x_{1}, x_{2}, \ldots, x_{N}\right)=0 \\
f_{2}\left(x_{1}, x_{2}, \ldots, x_{N}\right)=0 \\
\cdot \cdot \\
f_{N}\left(x_{1}, x_{2}, \ldots, x_{N}\right)=0
\end{array}\right\} \quad \text { or } \quad F(X)=0
$$

While $\mathrm{X}$ is vector for $\mathrm{n}$ unknown state variables, and $\mathrm{F}$ stands for the set of $\mathrm{n}$ nonlinear equations.

In principle, through executing the expansion of Taylor series for $\mathrm{F}(\mathrm{X})$ around the initial assumption $\mathrm{X}(0)$, the vector of state variables $\mathrm{X}$ can be determined. This is in brief what the methodology comprises of. Taylor expansion is shown below:

$$
F(X)=F\left(X^{(0)}\right)+J\left(X^{(0)}\right)\left(X-X^{(0)}\right)+\text { higher-order terms }
$$

$\mathrm{J}\left(\mathrm{X}^{(0)}\right)$ will be called Jacobian matrix. This represents the matrix of first-order partial derivatives for $\mathrm{F}(\mathrm{X})$ with respect to $\mathrm{X}$, calculated at $\mathrm{X}=\mathrm{X}^{(0)}$.

By presuming that $\mathrm{X}^{(1)}$ represents the value calculated through method for iteration 1 and it will be adequately near to primary guess $\mathrm{X}^{(0)}$, the expansion leads towards an appropriate calculation to compute the vector of state variables $\mathrm{X}$. Based upon this proposition, all high-order derivative expressions in Eq. (15) can be ignored. Thus,

$$
\left[\begin{array}{c}
\mathrm{f}_{1}\left(\mathrm{X}^{(1)}\right) \\
\mathrm{f}_{2}\left(\mathrm{X}^{(1)}\right) \\
\cdot \\
\cdot \\
\cdot \\
\mathrm{f}_{n}\left(\mathrm{X}^{(1)}\right)
\end{array}\right] \approx\left[\begin{array}{c}
\mathrm{f}_{1}\left(\mathrm{X}^{(0)}\right) \\
\mathrm{f}_{2}\left(\mathrm{X}^{(0)}\right) \\
\cdot \\
\cdot \\
\mathrm{f}_{n}\left(\mathrm{X}^{(0)}\right)
\end{array}\right]+\left[\begin{array}{cccc}
\frac{\partial \mathrm{f}_{1}(\mathrm{X})}{\partial x_{1}} & \frac{\partial \mathrm{f}_{1}(\mathrm{X})}{\partial x_{2}} & \cdots & \frac{\partial \mathrm{f}_{1}(\mathrm{X})}{\partial x_{n}} \\
\frac{\partial \mathrm{f}_{2}(\mathrm{X})}{\partial x_{1}} & \frac{\partial \mathrm{f}_{2}(\mathrm{X})}{\partial x_{2}} & & \frac{\partial \mathrm{f}_{2}(\mathrm{X})}{\partial x_{n}} \\
\vdots & & \ddots & \vdots \\
\frac{\partial \mathrm{f}_{n}(\mathrm{X})}{\partial x_{1}} & \frac{\partial \mathrm{f}_{n}(\mathrm{X})}{\partial x_{2}} & \cdots & \frac{\partial \mathrm{f}_{n}(\mathrm{X})}{\partial x_{n}}
\end{array}\right]\left[\begin{array}{c}
X_{1}^{(1)}-X_{1}^{(0)} \\
X_{2}^{(1)}-X_{2}^{(0)} \\
\cdot \\
\cdot \\
\cdot \\
X_{n}^{(1)}-X_{n}^{(0)}
\end{array}\right]
$$

In case for iteration (i), we generalize the above expression in compact form as,

$$
\mathrm{F}\left(\mathrm{X}^{(i)}\right) \approx \mathrm{F}\left(\mathrm{X}^{(i-1)}\right)+\mathrm{J}\left(\mathrm{X}^{(i-1)}\right)\left(\mathrm{X}^{(i)}-\mathrm{X}^{(i-1)}\right)
$$

Where $\mathrm{i}=1,2 \ldots$ Moreover in case, this has been supposed that $X^{(i)}$ is adequately near to result $\mathrm{X}^{(*)}$ then $F\left(X^{(i)}\right) \approx F\left(X^{(*)}\right)=$ 0 . Thus, Eq. (13) transforms to

$$
F\left(X^{(i-1)}\right)+J\left(X^{(i-1)}\right)\left(X^{(i)}-X^{(i-1)}\right)=0
$$


Furthermore, resolving for $\mathrm{X}^{(\mathrm{i})}$,

$$
X^{(i)}=X^{(i-1)}-J^{-1}\left(X^{(i-1)}\right) F\left(X^{(i-1)}\right)
$$

Iterative result may formulate to be the function for correction vector $\triangle X^{(i)}=X^{(i)}-\triangle X^{(i-1)}$,

$$
\Delta X^{(i)}=-J^{-1}\left(X^{(i-1)}\right) F\left(X^{(i-1)}\right)
$$

and initial assumptions are upgraded employing the equation below:

$$
X^{(i)}=X^{(i-1)}+\triangle X^{(i)}
$$

Employing the most recent values of $\mathrm{X}$ in Eq. (20), the calculations are performed again and again as many times as needed. It is executed till mismatches $\Delta \mathrm{X}$ are inside the specified small tolerance $\left(10^{-12}\right)$.

For finding solution of load flow problem and applying NR methodology, Eq. (20) represents all the related equations, where $\mathrm{X}$ stands for the set of unknown phase angles and bus voltage magnitudes. The expansions about the base term $(\theta(0), \mathrm{V}(0))$ is performed for mismatch equations $\Delta \mathrm{P}$ and $\Delta \mathrm{Q}$ and, thus, the following relationship is formed for the load flow Newton Raphson method:

$$
\left[\begin{array}{l}
\triangle P \\
\triangle Q
\end{array}\right]=-\left[\begin{array}{ll}
\frac{\partial P}{\partial \theta} & \frac{\partial P}{\partial V} V \\
\frac{\partial Q}{\partial \theta} & \frac{\partial Q}{\partial V} V
\end{array}\right]\left[\begin{array}{l}
\triangle \theta \\
\frac{\Delta V}{V}
\end{array}\right]
$$

Various matrices of Jacobian can comprise up to $\left(\mathrm{n}_{b}-1\right) \times\left(\mathrm{n}_{b}-1\right)$ entries:

$$
\left.\begin{array}{ll}
\frac{\partial P_{k}}{\partial \theta_{m}}, & \frac{\partial P_{k}}{\partial V_{m}} V_{m} \\
\frac{\partial Q_{k}}{\partial \theta_{m}}, & \frac{\partial Q_{k}}{\partial V_{m}} V_{m}
\end{array}\right\}
$$

and $\mathrm{k}=1, \ldots, \mathrm{n}_{b}$, and $\mathrm{m}=1, \ldots, \mathrm{n}_{b}$ however, eliminating slack bus elements.

Voltage magnitude $\mathrm{V}$ and reactive power Q are also omitted for PV buses for the related rows and columns. Moreover, the related $\mathrm{k}-\mathrm{m}$ element in Jacobian matrix is zero in case bus $\mathrm{k}$ and bus $\mathrm{m}$ don't directly associate through a transmission element. Jacobians of power-flows are largely scarce because of slow rate connection that is common for power networks. An extra characteristic is that they are symmetrical in shape but not in value.

In order to recompense for the feature that Jacobian expressions $\left(\partial P_{k} / \partial V_{m}\right) V_{m}$ and $\left(\partial Q_{k} / \partial V_{m}\right) V_{m}$ are multiplied by $\mathrm{V}_{m}$, it should be indicated that correction expressions $\Delta \mathrm{V}_{m}$ have been divided by $\mathrm{V}_{m}$. This assumption leads towards very helpful and simple results as represented in the derivative expressions shown below.

Let an $\mathrm{l}^{\text {th }}$ element be linked with bus $\mathrm{k}$ and bus $\mathrm{m}$, for which mutual and self Jacobian expressions have been shown beneath: For $\mathrm{k} \neq \mathrm{m}$ :

$$
\begin{gathered}
\frac{\partial P_{k, l}}{\partial \theta_{m, l}}=V_{k} V_{m}\left[G_{k m} \sin \left(\theta_{k}-\theta_{m}\right)-B_{k m} \cos \left(\theta_{k}-\theta_{m}\right)\right] \\
\frac{\partial P_{k, l}}{\partial V_{m, l}} V_{m, l}=V_{k} V_{m}\left[G_{k m} \cos \left(\theta_{k}-\theta_{m}\right)-B_{k m} \sin \left(\theta_{k}-\theta_{m}\right)\right] \\
\frac{\partial Q_{k, l}}{\partial \theta_{m, l}}=-\frac{\partial P_{k, l}}{\partial V_{m, l}} V_{m, l} \\
\frac{\partial Q_{k, l}}{\partial V_{m, l}} V_{m, l}=\frac{\partial P_{k, l}}{\partial \theta_{m, l}}
\end{gathered}
$$


For $k=m$ :

$$
\begin{gathered}
\frac{\partial P_{k, l}}{\partial \theta_{m, l}}=-Q_{k}^{c a l}-V_{k}^{2} B_{k k} \\
\frac{\partial P_{k, l}}{\partial V_{m, l}} V_{k, l}=P_{k}^{c a l}+V_{k}^{2} G_{k k} \\
\frac{\partial Q_{k, l}}{\partial \theta_{m, l}}=P_{k}^{c a l}-V_{k}^{2} G_{k k} \\
\frac{\partial Q_{k, l}}{\partial V_{m, l}} V_{k, l}=Q_{k}^{c a l}-V_{k}^{2} B_{k k}
\end{gathered}
$$

Generally, the bus self-elements transform to the following format for the bus $\mathrm{k}$ comprising $\mathrm{n}$ transmission elements $\mathrm{l}$ :

$$
\begin{gathered}
\frac{\partial P_{k}}{\partial \theta_{k}}=\sum_{l=1}^{n} \frac{\partial P_{k, l}}{\partial \theta_{k, l}} \\
\frac{\partial P_{k}}{\partial V_{k}} V_{k}=\sum_{l=1}^{n} \frac{\partial P_{k, l}}{\partial V_{k, l}} V_{k, l} \\
\frac{\partial Q_{k}}{\partial \theta_{k}}=\sum_{l=1}^{n} \frac{\partial Q_{k, l}}{\partial \theta_{k, l}} \\
\frac{\partial Q_{k}}{\partial V_{k}} V_{k}=\sum_{l=1}^{n} \frac{\partial Q_{k, l}}{\partial V_{k, l}} V_{k, l}
\end{gathered}
$$

Whether there are $\mathrm{n}$ transmission elements or one transmission element finishing on bus $\mathrm{k}$, mutual elements presented in Eqs. (24) and (27) stand the same. The reactive and active load-flows all over transmission network will be calculated very simply after the phase angles and voltage magnitudes have been computed by iteration. A significant point to keep in mind is that the yet to be found variables $\mathrm{P}_{\text {slack }}$ and $\mathrm{Q}_{\text {slack }}$ will be calculated after network load-flows and the mismatch expressions $\Delta \mathrm{P}$ and $\Delta \mathrm{Q}$ for swing bus haven't been added to Eq. (22) since the computations are carried out for n-1 buses excluding the swing or slack bus.. Also, to find out whether the generators are inside reactive power limits, QG for PV nodes will be computed during every cycle. But mismatch reactive power expressions $\Delta \mathrm{Q}$ of PV nodes won't be added to Eq. (22).

\subsection{Newton Raphson Method for power flow solution}

In this technique, we have two solution techniques available for power flow result. One is rectangular coordinate method and the other is polar coordinate method. We will use polar coordinate method as it is the most widely used method.

The complex power in bus i can be expressed through Eq. (36) while Eqs. (38) and (39) give active and reactive powers:

$$
\begin{gathered}
S_{i}=P_{i}+j Q_{i}=V_{i} \sum_{k=1}^{n} Y_{i k}^{*} V_{k}^{*} \\
S_{i}=\sum_{k=1}^{n}\left(V_{i} V_{k} Y_{i k}\right) \angle\left(\delta_{i}-\delta_{k}-\theta_{i k}\right) \\
P_{i}=\sum_{k=1}^{n}\left(V_{i} V_{k} Y_{i k}\right) \cos \left(\delta_{i}-\delta_{k}-\theta_{i k}\right)
\end{gathered}
$$




$$
Q_{i}=\sum_{k=1}^{n}\left(V_{i} V_{k} Y_{i k}\right) \sin \left(\delta_{i}-\delta_{k}-\theta_{i k}\right)
$$

We can also write Eqs. (38) and (39) as:

$$
\begin{gathered}
P_{i}=V_{i} V_{i} Y_{i i} \cos \theta_{i i}+\sum_{\substack{k=1 \\
k \neq i}}^{n}\left(V_{i} V_{k} Y_{i k}\right) \cos \left(\delta_{i}-\delta_{k}-\theta_{i k}\right) \\
Q_{i}=V_{i} V_{i} Y_{i i} \cos \theta_{i i}+\sum_{\substack{k=1 \\
k \neq i}}^{n}\left(V_{i} V_{k} Y_{i k}\right) \sin \left(\delta_{i}-\delta_{k}-\theta_{i k}\right)
\end{gathered}
$$

And $\triangle f=J \triangle X$

$$
\text { If } \quad \Delta P_{i}=P_{i(s p)}-P_{i(c a l)}
$$

Then $i=1,2, \cdots, n \quad i \neq$ slack

$$
\text { If } \quad \Delta Q_{i}=Q_{i(s p)}-Q_{i(c a l)}
$$

Then $i=1,2, \cdots, n \quad i \neq$ slack $\quad i \neq$ PVbus

In Eq. (42), the subscripts sp and cal represent respectively, specified and calculated values. And

$$
\left[\begin{array}{l}
\triangle P \\
\triangle Q
\end{array}\right]=\left[\begin{array}{cc}
H & N \\
M & L
\end{array}\right]\left[\begin{array}{c}
\triangle \delta \\
\triangle V
\end{array}\right]
$$

$\mathrm{H}, \mathrm{N}, \mathrm{M}$ and $\mathrm{L}$ are sub-matrices which are found by differentiating with respect to $\delta$ and $|\mathrm{V}|$ Eqs. (38) and (39). We calculate off-diagonal elements of $\mathrm{H}$ by

$$
H_{i k} \triangleq \frac{\partial P_{i}}{\partial \delta_{k}}=V_{i} V_{k} Y_{i k} \sin \left(\delta_{i}-\delta_{k}-\theta_{i k}\right), \quad i \neq k
$$

While for diagonal entries:

$$
H_{i i} \triangleq \frac{\partial P_{i}}{\partial \delta_{i}}=-V_{i} \sum_{\substack{k=1 \\ k \neq i}}^{n} Y_{i k} V_{k} \sin \left(\delta_{i}-\delta_{k}-\theta_{i}\right)
$$

From Eq. (40)

$$
H_{i i}=-Q_{i}-B_{i i} V_{i}^{2}
$$

Similarly, the diagonal expressions for $\mathrm{N}$ will be:

$$
\frac{\partial P_{i}}{\partial\left|V_{i}\right|}=2 V_{i} Y_{i i} \cos \theta_{i i}+\sum_{\substack{k=1 \\ k \neq i}}^{n} Y_{i k} V_{k} \cos \left(\delta_{i}-\delta_{k}-\theta_{i k}\right)
$$

and off-diagonal elements

$$
\frac{\partial P_{i}}{\partial\left|V_{k}\right|}=V_{i} V_{k} \cos \left(\delta_{i}-\delta_{k}-\theta_{i k}\right)
$$

Likewise, off-diagonal and diagonal expressions for $\mathrm{M}$ will be

$$
\frac{\partial Q_{i}}{\partial \delta_{k}}=-V_{i} V_{k} Y_{i k} \cos \left(\delta_{i}-\delta_{k}-\theta_{i k}\right), \quad i \neq k
$$




$$
\frac{\partial Q_{i}}{\partial \delta_{i}}=\sum_{\substack{k=1 \\ k \neq i}}^{n} V_{i} V_{k} Y_{i k} \cos \left(\delta_{i}-\delta_{k}-\theta_{i k}\right)
$$

And off-diagonal and diagonal expressions for $\mathrm{L}$ will be

$$
\begin{gathered}
\frac{\partial Q_{i}}{\partial V_{k}}=V_{i} Y_{i k} \sin \left(\delta_{i}-\delta_{k}-\theta_{i k}\right), \quad i \neq k \\
\frac{\partial Q_{i}}{\partial V_{i}}=-2 V_{i} Y_{i i} \sin \theta_{i i}+\sum_{\substack{k=1 \\
k \neq i}}^{n} V_{k} Y_{i k} \sin \left(\delta_{i}-\delta_{k}-\theta_{i k}\right)
\end{gathered}
$$

Updated voltage magnitude and angle for next iteration are computed as below:

$$
\begin{gathered}
\left|V_{i}^{(r+1)}\right|=\left|V_{i}^{(r)}\right|+\triangle\left|V_{i}^{(r)}\right| \\
\delta_{i}^{(r+1)}=\delta_{i}^{(r)}+\triangle \delta_{i}^{(r)}
\end{gathered}
$$

Where subscript $\mathrm{r}$ represents values from previous iteration and subscript $\mathrm{r}+1$ represents the next iteration values.

\section{Results and discussion}

This section is divided into two subsections. First subsection describes the data while the second one gives the results. The network is as shown in figure below:

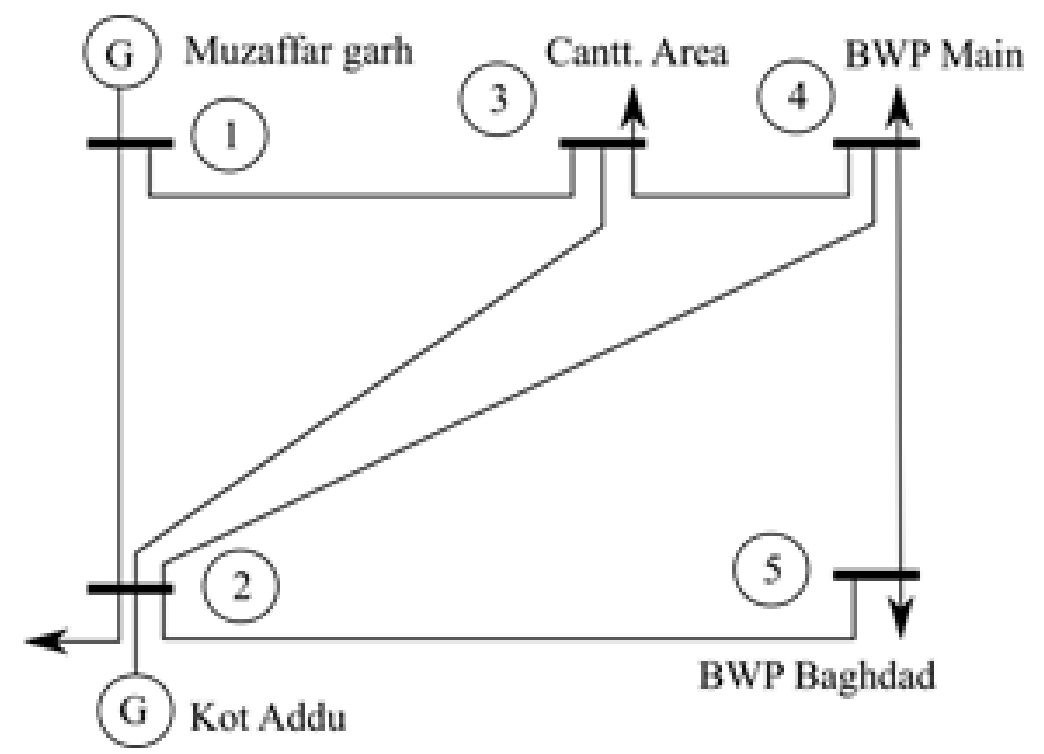

Fig 2. The Five-bus Network in Bahawalpur Area

\subsection{Power flow data}

The data of the above network is classified into four subsections. It constitutes bus specifications, generator power limits, transmission line impedances and admittances and lastly, the load descriptions at various buses. Literature and field survey is conducted to collect power flow data of the selected area. All the aforementioned parameters will be expounded in detail one by one. 


\subsubsection{Bus data}

The five buses in system illustrated in Figure 2 are numbered for identification in load flow algorithm. The type of each bus is mentioned next which is followed by the voltages (magnitude and angle) of the respective buses. Voltage magnitude is expressed in per unit (p.u.) and phase angle is given in degrees. The Muzaffargarh generator bus is chosen as the slack bus and voltage magnitude $\mathrm{V}_{\text {slack }}$ and phase angle $\theta_{\text {slack }}$, are specified. The phase angle $\theta_{\text {slack }}$ is selected as reference against the rest of the angles. It is evident that the voltage at each bus is approximately the same. All of these bus parameters are shown in Table 1 .

Table 1. Bus-data for the network under consideration.

\begin{tabular}{|c|c|c|c|}
\hline Bus & Number & Type & Voltage \\
\hline Muzaffargarh & 1 & Slack & $1.00 \angle 0$ \\
\hline Kot Addu & 2 & Generator & $1.00 \angle 0$ \\
\hline Cantt. Area & 3 & Load & $1.00 \angle 0$ \\
\hline BWP Main & 4 & Load & $1.00 \angle 0$ \\
\hline BWP Baghdad & 5 & Load & $1.00 \angle 0$ \\
\hline
\end{tabular}

\subsubsection{Generator data}

The scheduled active power PGEN and reactive power QGEN contributed by the two generator buses are shown below along with the upper and lower limits of reactive power QMAX and QMIN respectively. This data is shown in Table 2.

Table 2. Generator-data for the network under consideration.

\begin{tabular}{llll}
\hline \multirow{2}{*}{ Bus } & \multirow{2}{*}{ Power Generated PQ $\mathbf{S E N}$} & \multicolumn{2}{c}{ Reactive Power Limit } \\
\cline { 3 - 4 } & & ${\text { Upper Limit } \mathbf{Q}_{M A X}}^{\text {Lower Limit }_{M I N}}$ \\
\hline Muzaffargarh & $0+0 i$ & 8 & -8 \\
Kot Addu & $0.16+0 i$ & 5 & -5 \\
\hline
\end{tabular}

\subsubsection{Transmission line data}

The relation between the transmission line number and the bus number is elucidated below as shown in Figure 2 also. It is elucidated that the transmission line 1 connects buses 1 and 2, transmission line 2 connects buses 1 and 3 and similarly, the rest of the transmission lines are attached as tabularized below. This is followed by the impedances of the respective transmission lines, here $\mathrm{Z}$ stands for the series impedance which is the sum of series resistance $\mathrm{R}$ and series reactance $\mathrm{X}$. In the last column, admittances of the corresponding transmission lines are represented, where the shunt admittance $\mathrm{Y}$ is the sum of shunt conductance $G$ and shunt susceptance $B$. The data is expressed in Table 3.

Table 3. Line-data for the network under consideration.

\begin{tabular}{llll}
\hline Bus Number & Line Number & Series Impedance $(Z=R+X)$ & Shunt Admittance $(\mathbf{Y}=\mathbf{G}+\mathbf{B})$ \\
\hline $1-2$ & 1 & $0.020579+0.052057$ & $0+0.06 i$ \\
$1-3$ & 2 & $0.0198017+0.1294047 i$ & $0+0.05 i$ \\
$2-3$ & 3 & $0.006332+0.016017 i$ & $0+0.04 i$ \\
$2-4$ & 4 & $0.006901+0.045095 i$ & $0+0.04 i$ \\
$2-5$ & 5 & $0.0216018+0.14116 i$ & $0+0.03 i$ \\
$3-4$ & 6 & $0.052241+0.132146 i$ & $0+0.02 i$ \\
$4-5$ & 7 & $0.020579+0.052057 i$ & $0+0.05 i$ \\
\hline
\end{tabular}

\subsubsection{Load data}

The scheduled real power $\mathrm{P}_{L O A D}$ and scheduled reactive power $\mathrm{Q}_{L O A D}$ consumed at load buses will be as shown in Table 4 . 
Table 4. Load-datafor the network under consideration

\begin{tabular}{ll}
\hline Bus & Power Consumed PQ $L O A D$ \\
\hline Kot Addu & $0.226+0.1094 i$ \\
Cantt. Area & $0.168+0.0812 i$ \\
BWP Main & $0.189+0.091 i$ \\
Baghdad & $0.104+0.0508 i$ \\
\hline
\end{tabular}

The network employed to show load flow results provided through NR and FD approaches, is from Bahawalpur area as shown in the Figure 2. It represents the five-bus system consisting of two generator uses and three load buses including seven transmission lines. One generator bus is from Muzaffargarh and the other bus is situated in Kot Addu around Bahawalpur. The three load buses are at Cantt. Area, BWP Main and BWP Baghdad respectively. The data at length is given in the following section and is feasible to utilize either with NR or FD programs.

\subsubsection{Bus voltage results}

The bus voltage magnitude is expressed in per unit (p.u.) and phase angle in degrees against their respective buses. The results are elaborated in Table 5 .

Table 5. Bus voltage for the network under consideration.

\begin{tabular}{lll}
\hline \multirow{2}{*}{ Bus } & \multicolumn{2}{c}{ Voltage } \\
\cline { 2 - 3 } & Magnitude & Angle \\
\hline Muzaffargarh & 1.00 & 0.00 \\
Kot Addu & 1.00 & -1.2425 \\
Cantt. Area & 0.9990 & -1.2735 \\
BWP Main & 0.9970 & -1.6759 \\
BWP Baghdad & 0.9964 & -1.7847 \\
\hline
\end{tabular}

\subsubsection{Power flow results}

The sending end real power $\mathrm{P}_{S E N D}$ and reactive power $\mathrm{Q}_{S E N D}$ have been catalogued below followed by the receiving end real power $\mathrm{P}_{R E C}$ and reactive power $\mathrm{Q}_{R E C}$ against their respective transmission lines. The buses associated with lines are also tabularized. These power flow results have been presented in Table 6 .

Table 6. Power flow results for the network under consideration.

\begin{tabular}{|c|c|c|c|}
\hline Bus Number & Line Number & Sending End Power PQ $S E N D$ & Receiving End Power PQ $R E C$ \\
\hline $1-2$ & 1 & $0.3618-0.1685 i$ & $-0.3587+0.1163 i$ \\
\hline $1-3$ & 2 & $0.1691-0.0414 i$ & $-0.1685-0.0049 i$ \\
\hline $2-3$ & 3 & $0.0502+0.0216 i$ & $-0.0502-0.0615 i$ \\
\hline $2-4$ & 4 & $0.1734+0.0204 i$ & $-0.1732-0.0588 i$ \\
\hline $2-5$ & 5 & $0.0691-0.0001 i$ & $-0.0690-0.0291 i$ \\
\hline $3-4$ & 6 & $0.0510-0.0148 i$ & $-0.0509-0.0048 i$ \\
\hline $4-5$ & 7 & $0.0351-0.0279 i$ & $-0.0350-0.0217 i$ \\
\hline
\end{tabular}

\section{Conclusion}

In this research, Newton Raphson MATLAB algorithm is developed for a ring distribution network of five-buses which is feeding different areas of Bahawalpur to find the solution for load flow problem. Power flow solutions provide systematic approach to calculate voltage magnitude, phase angle, active and reactive power before they occur in generation, load, transmission lines or interconnections ${ }^{(25)}$. Furthermore, power flow study is essentially important to estimate the reactive power which is a salient feature of power systems with widespread penetration of inductive loads and their compensation using FACTS devices ${ }^{(26)}$. Literature and field survey is conducted to collect power flow data of the selected area and the load flow equations of primarily Newton Raphson algorithm and Fast-decoupled method secondarily. Consequently, this data is utilized in the above mentioned 
algorithms to find out the optimum solution, best operation of the existing system and planning for future expansion to be at par with the growing demand.

\section{References}

1) Elgerd OI, Happ HH. Electric Energy Systems Theory: An Introduction. IEEE Transactions on Systems, Man, and Cybernetics. 1972;SMC-2(2):296-297. Available from: https://dx.doi.org/10.1109/tsmc.1972.4309116.

2) Tinney W, Hart C. Power Flow Solution by Newton's Method. IEEE Transactions on Power Apparatus and Systems. 1967;PAS-86(11):1449-1460. Available from: https://dx.doi.org/10.1109/tpas.1967.291823.

3) Stott B, Alsac O. Fast Decoupled Load Flow. IEEE Transactions on Power Apparatus and Systems. 1974;PAS-93(3):859-869. Available from: https: //dx.doi.org/10.1109/tpas.1974.293985.

4) Zhang F, Cheng CS. A modified Newton method for radial distribution system power flow analysis. IEEE Transactions on Power Systems. 1997;12(1):389397.

5) Jasmon GB, Lee LHCC. Distribution network reduction for voltage stability analysis and loadflow calculations. International Journal of Electrical Power \& Energy Systems. 1991;13(1):9-13. Available from: https://dx.doi.org/10.1016/0142-0615(91)90011-j.

6) Chen TH, Chen MS, Hwang KJ, Kotas P, Chebli EA. Distribution system power flow analysis-a rigid approach. IEEE Transactions on Power Delivery. 1991;6(3):1146-1152. Available from: https://dx.doi.org/10.1109/61.85860.

7) Lin WM, Teng JH. Three-phase distribution network fast-decoupled power flow solutions. International Journal of Electrical Power \& Energy Systems. 2000;22(5):375-380. Available from: https://dx.doi.org/10.1016/s0142-0615(00)00002-8.

8) Teng JH. A modified Gauss-Seidel algorithm of three-phase power flow analysis in distribution networks. International Journal of Electrical Power and Energy Systems. 2000;24(2). Available from: https://doi.org/10.1016/S0142-0615(01)00022-9.

9) Tripathy SC, Prasad G, Malik OP, Hope GS. Load-Flow Solutions for Ill-Conditioned Power Systems by a Newton-Like Method. IEEE Transactions on Power Apparatus and Systems. 1982;PAS-101(10):3648-3657. Available from: https://dx.doi.org/10.1109/tpas.1982.317050.

10) Baran M, Wu FF. Optimal sizing of capacitors placed on a radial distribution system. IEEE Transactions on Power Delivery. 1989;4(1):735-743. Available from: https://dx.doi.org/10.1109/61.19266.

11) Chiang HD. A decoupled load flow method for distribution power network algorithms, analysis and convergence study. International Journal of Electrical Power and Energy Systems. 1991;13(3):130-138. Available from: https://doi.org/10.1016/0142-0615(91)90001-C.

12) Goswami SK, Basu SK. Direct solution of distribution systems. IEE Proceedings C Generation, Transmission and Distribution. 1991;138(1):78-78. Available from: https://dx.doi.org/10.1049/ip-c.1991.0010.

13) Jasmon GB, Lee LHCC. Distribution network reduction for voltage stability analysis and loadflow calculations. International Journal of Electrical Power \& Energy Systems. 1991;13(1):9-13. Available from: https://dx.doi.org/10.1016/0142-0615(91)90011-j.

14) Das D, Nagi HS, Kothari DP. Novel method for solving radial distribution networks. In: and others, editor. IEE Proceedings on Generation Transmission and Distribution;vol. 141 of 4. 1994;p. 291-298. Available from: https://doi.org/10.1049/ip-gtd:19949966.

15) Haque M. Efficient load-flow method for distribution systems with radial or mesh configuration. IEE Proceedings on Generation, Transmission, Distribution. 1996;143(1):33-39.

16) Eminoglu U, Hocaoglu MH. A new power flow method for radial distribution systems including voltage dependent load models. Electric Power Systems Research. 2005;76:106-114. Available from: https://dx.doi.org/10.1016/j.epsr.2005.05.008.

17) Prasad K, Sahoo NC, Chaturvedi A, Ranjan R. A Simple Approach for Branch Current Computation in Load Flow Analysis of Radial Distribution Systems. International Journal of Electrical Engineering \& Education. 2007;44(1):49-63. Available from: https://dx.doi.org/10.7227/ijeee.44.1.6.

18) Ghosh S, Sherpa K. An efficient method for load-flow solution of radial distribution networks. International Journal of Electrical and Computer Engineering. 2008;2(9):2094-2101.

19) Sivanagaraju S, Rao JV, Giridhar M. A loop based load flow method for weakly meshed distribution network. ARPN Journal of Engineering and Applied Sciences. 2008;3(4):55-59.

20) Kumar A, Aravindhababu P. An improved power flow technique for distribution systems. Journal of Computer Science, Informatics and Electrical Engineering. 2009;3(1).

21) Augugliaro A, Dusonchet L, Favuzza S, Ippolito MG, Sanseverino ER. A backward sweep method for power flow solution in distribution networks. International Journal of Electrical Power \& Energy Systems. 2010;32(4):271-280. Available from: https://dx.doi.org/10.1016/j.ijepes.2009.09.007.

22) Kaur G. Load-flow analysis of radial distribution networks. 2012.

23) Sharma DP, Chaturvedi A, Purohit G, Prasad G. An improved mechanism of a leaf node identification for radial distribution network. IEEE Power and Energy Conference at Illinois. 2011. Available from: https://doi.org/10.1109/PECI.2011.5740477.

24) Parasher R. Load flow analysis of radial distribution network using linear data structure. 2013. Available from: https://arxiv.org/ftp/arxiv/papers/1403/ 1403.4702.pdf.

25) Afolabi OA, Ali WH, Cofie P, Fuller J, Obiomon P, Kolawole ES. Analysis of the Load Flow Problem in Power System Planning Studies. Energy and Power Engineering. 2015;07(10):509-523. Available from: https://dx.doi.org/10.4236/epe.2015.710048.

26) AbdElhafez AA, Alruways SH, Alsaif YA, Althobaiti MF, AlOtaibi AB, Alotaibi NA. Reactive Power Problem and Solutions: An Overview. Journal of Power and Energy Engineering. 2017;05(05):40-54. Available from: https://dx.doi.org/10.4236/jpee.2017.55004. 